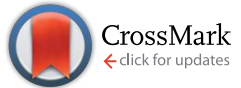

Cite this: J. Mater. Chem. A, 2016, 4, 15676

Received 20th August 2016

Accepted 12th September 2016

DOI: $10.1039 / c 6 t a 07149 j$

www.rsc.org/MaterialsA

\section{Highly efficient electrochemical and chemical hydrogenation of 4-nitrophenol using recyclable narrow mesoporous magnetic CoPt nanowires $\dagger$}

\begin{abstract}
Albert Serrà, ${ }^{a}$ Xavier Alcobé, ${ }^{b}$ Jordi Sort, ${ }^{\text {cd }}$ Josep Nogués ${ }^{\text {ed }}$ and Elisa Vallés ${ }^{\star a}$
Toxic nitro-compounds, such as 4-nitrophenol, are one of the most common wastewater industrial pollutants. Thus, efficient ways to neutralize them are actively pursued. Here novel procedures to degrade these types of compounds based on the use of mesoporous magnetic nanowires are demonstrated. Fully-mesoporous magnetic narrow $(25 \mathrm{~nm})$ CoPt nanowires with an extraordinary effective area are grown using ionic liquid-in-water microemulsions in alumina templates. These mesoporous nanowires are shown to be efficient catalysts for the hydrogenation of 4-nitrophenol by electrocatalysis. Additionally, these nanowires also present exceptional conventional catalytic activity when used in conjunction with $\mathrm{NaBH}_{4}$, particularly when magnetic stirring is utilized. In fact, magnetically actuated mesoporous CoPt nanowires drastically outperform all state-of-the-art 4-nitrophenol catalysts. Additionally, given their magnetic character, these nanowires can be easily recycled and reused. Thus, the outstanding catalytic performance of mesoporous CoPt nanowires makes them excellent candidates for wastewater treatment agents.
\end{abstract}

\section{Introduction}

Aromatic nitro-compounds such as nitrobenzene, nitrophenols and nitrotoluenes, which have considerable toxic effects on humans, animals and plants, even at very low concentrations, are rather common in natural water. ${ }^{1-4}$ Most of these compounds have been included as hazardous chemicals in the environmental legislation of many countries. ${ }^{5,6}$ In fact, it is well known that nitrophenol and its derivatives are among the most refractory pollutants in industrial wastewater as resulting from the production process of insecticides, synthetic dyes or herbicides. ${ }^{7-9}$ Additionally, the conversion of aromatic nitro-compounds, especially with functionalized groups, into anilines ranks among the most important reactions in organic chemistry due to their broad range of applications, since they are key intermediates in the manufacturing of pharmaceuticals,

${ }^{a}$ Grup d'Electrodeposició de Capes Primes i Nanoestructures (GE-CPN), Departament de Ciència de Materials $i$ Química Física, Institut de Nanociència i Nanotecnologia (IN $\left.{ }^{2} U B\right)$, Universitat de Barcelona, Martí i Franquès, 1, E-08028, Barcelona, Catalonia, Spain.E-mail: e.valles@ub.edu

${ }^{b}$ Unitat de DRX, Centres Cientifics $i$ Tecnològics, Universitat de Barcelona, CCiTUB, Lluís Solé i Sabarís, 1-3, E-08028, Barcelona, Catalonia, Spain

${ }^{c}$ Departament de Física, Universitat Autònoma de Barcelona, E-08193, Bellaterra, Catalonia, Spain

${ }^{d}$ ICREA, Pg. Lluís Companys 23, 08010, Barcelona, Spain

${ }^{e}$ Catalan Institute of Nanoscience and Nanotechnology (ICN2), CSIC, The Barcelona Institute of Science and Technology, Campus UAB, E-08193, Bellaterra, Spain

$\uparrow$ Electronic supplementary information (ESI) available. See DOI: $10.1039 /$ c6ta07149j agrochemicals, dyes and pigments. ${ }^{\mathbf{1 0 - 1 3}}$ Nowadays, to neutralize these harmful chemicals, non-catalytic reductions with reducing agents such as sulphides and Fe are used. Unfortunately, these processes generate large amounts of residues. ${ }^{\mathbf{1 4 - 1 6}}$ Compared with those conversion routes, catalytic reduction using sodium borohydride as a reducing agent in aqueous media is a relatively simple and clean method. ${ }^{17-19}$ However, the efficacy of this type of approach still remains moderate. Hence, there is a strong need to develop more efficient, inexpensive and environmentally friendly catalytic alternatives to convert 4-nitrophenol (4-NP) to 4-aminophenol (4-AP) with high catalytic activity and good chemoselectivity.

Interestingly, Pt-group metals present high catalytic activity for hydrogenation of nitro-compounds due to their high ability for $\mathrm{H}_{2}$ generation. Nevertheless, they show poor chemoselectivity. ${ }^{20-22}$ However, chemoselectivity can be enhanced with the aid of transition metals. It is actually well known that cobalt or cobalt and iron oxide-based catalysts deliver excellent chemoselectivity for hydrogenation reactions. ${ }^{23-25}$ Therefore, the development of Co-Pt catalysts could be interesting for the hydrogenation of 4-nitrophenol given their potential to increase the activity and selectivity for these reactions. Moreover, Co-Pt alloys also exhibit tuneable magnetic properties depending on their composition, which allow them to be remotely actuated by means of external magnetic fields. This provides an excellent strategy to recover and recycle catalysts for iterative uses or to guide them to specific locations within the liquid. ${ }^{26-28}$

It is worth highlighting that nanostructuring, aiming at enhancing the surface area-to-volume ratio, has emerged in 
recent years as an advantageous tool to boost the activity of catalysts. Particularly, open-cell porous and especially mesoporous (i.e., with pore sizes ranging between 2 and $50 \mathrm{~nm}$ ) nanostructures, have rapidly developed as potential catalysts due to their low density, high surface area, and presence of large amounts of interstitial hollow cavities in the interconnected porous network. ${ }^{29-31}$ Hence, within this framework, the development of CoPt mesoporous nanostructures could be a promising way to improve the yield in the catalysis of the hydrogenation of 4-NP to 4-AP.

Over the last few years, the formation of well-defined mesoporous metallic nanostructures by means of the soft-templating method based on the use of liquid crystals or micellar solutions has been established as a promising fabrication strategy. ${ }^{32-34}$ Very recently, the use of microemulsions has been proposed as a general electrochemical pathway for the shape-controlled synthesis of mesoporous nanomaterials. ${ }^{35-38}$ However, due to the relatively high viscosity of the microemulsion, the electrochemical growth of mesoporous nanowires (NWs) inside the nanochannels of alumina or polycarbonate membranes remains challenging, given the difficulty of properly filling the narrow pores with the electrolyte.

Here, we first demonstrate the feasibility to grow magnetic mesoporous Co-Pt alloyed NWs with $25 \mathrm{~nm}$ diameter by electrodeposition using an ionic liquid-in-aqueous solution (IL/W) microemulsion. Then, we demonstrate that these mesoporous NWs show excellent performance during the electrocatalysis and catalysis for the hydrogenation of $4-\mathrm{NP}$, which is maintained after the NWs have been recycled and reused several times. Moreover, pure Co and Pt mesoporous NWs were also synthesised and tested in order to demonstrate the excellent catalytic performance of the mesoporous alloyed Co-Pt NWs as a consequence of their combined stability, magnetic behaviour, catalytic activity and chemoselectivity. Importantly, we show that magnetic actuation (magnetic stirring) can be used as an excellent tool to drastically boost the performance of the catalysis of 4-NP hydrogenation in both conventional catalysis and electrocatalysis. Hence, this simple novel procedure results in mesoporous CoPt NWs that remarkably outperform all state-of-the-art 4-NP hydrogenation catalysts.

\section{Experimental section}

\section{Electrochemical media}

The electrochemical media used to prepare NWs were:

(i) Compact nanowires. An aqueous solution $\left(\mathrm{W}_{1}\right)$ of $2.5 \mathrm{mM} \mathrm{CoCl}_{2} \cdot 6 \mathrm{H}_{2} \mathrm{O}$ (Carlo Erba, $\left.>98.0 \%\right)+1.2 \mathrm{mM} \mathrm{Na}_{2} \mathrm{PtCl}_{6} \cdot 6 \mathrm{H}_{2} \mathrm{O}$ (Alfa Aesar, 98\%) + 0.1 $\mathrm{M} \mathrm{NH}_{4} \mathrm{Cl}$ (Fluka, 99.5\%) + $10 \mathrm{~g} \mathrm{~L}^{-1} \mathrm{H}_{3} \mathrm{BO}_{3}$ (Merck, >98\%).

(ii) Mesoporous nanowires. Ionic-liquid in water $\left(\mathrm{IL} / \mathrm{W}_{i}\right)$ microemulsions, prepared by mixing the aqueous solution $\left(\mathrm{W}_{i}\right)$, surfactant (S) - p-octyl poly(ethyleneglycol) phenyl ether a.k.a. Triton X-100 (Acros Organics, 98\%) - and ionic liquid (IL) - 1butyl-3-methylimidazolium hexafluorophosphate a.k.a. bmimPF $_{6}$, $\mathrm{C}_{8} \mathrm{H}_{15} \mathrm{~F}_{6} \mathrm{~N}_{2} \mathrm{P}$, (Solvionic, 99\%) - in a 15.1 wt $\%$ of $\mathrm{S} / 1.1 \mathrm{wt} \%$ of the $\mathrm{IL} / 83.8 \%$ of the $\mathrm{W}_{i}$ proportion. ${ }^{35-39} \mathrm{Co}-\mathrm{Pt}$ mesoporous NWs were synthesised using the above-mentioned aqueous solution $\left(\mathrm{W}_{1}\right)$.
Alternatively, mesoporous NWs of pure Co and Pt were prepared using $\mathrm{W}_{2}-2.5 \mathrm{mM} \mathrm{CoCl} \cdot 6 \mathrm{H}_{2} \mathrm{O}$ (Carlo Erba, $\left.>98.0 \%\right)+0.1 \mathrm{M}$ $\mathrm{NH}_{4} \mathrm{Cl}$ (Fluka, 99.5\%) + $10 \mathrm{~g} \mathrm{~L}^{-1} \mathrm{H}_{3} \mathrm{BO}_{3}$ (Merck, >98\%) - and $\mathrm{W}_{3}-1.2 \mathrm{mM} \mathrm{Na}_{2} \mathrm{PtCl}_{6} \cdot 6 \mathrm{H}_{2} \mathrm{O}$ (Alfa Aesar, 98\%) $+0.1 \mathrm{M} \mathrm{NH} \mathrm{NH}_{4} \mathrm{Cl}$ (Fluka, 99.5\%) + $10 \mathrm{~g} \mathrm{~L}^{-1} \mathrm{H}_{3} \mathrm{BO}_{3}$ (Merck, >98\%), respectively. The mixture was stirred for 5 min under magnetic stirring (300 rpm) and argon bubbling, which prompted transparent, isotropic, thermodynamically stable microemulsions. All solutions were prepared with deionized water (Millipore Q-system) with a resistivity of $18.2 \mathrm{M} \Omega \mathrm{cm}$.

The physicochemical parameters for both aqueous solution and microemulsions were also determined. The electrical conductivity was measured using a Crison ${ }^{\mathrm{TM}}$ Conductimeter GLP31 with a 52-92 Crison ${ }^{\mathrm{TM}}$ conductivity cell $\left(1 \mathrm{~cm}^{-1}\right.$ of the cell constant). The viscosity measurements were performed using an Ostwald viscometer. Finally, the droplet size and polydispersity index of the microemulsion were analysed by dynamic light scattering (DLS) with a Zetasizer Nano ZS (Malvern $^{\mathrm{TM}}$ instruments). All the measurements were performed in triplicate.

\section{Electrochemical synthesis}

The electrochemical experiments were carried out using a microcomputer-controlled potentiostat/galvanostat Autolab with PGSTAT30 equipment and the GPES software. The electrodeposition process was performed at room temperature $\left(25^{\circ} \mathrm{C}\right)$, using a three-electrode electrochemical system, in potentiostatic mode. To define the NWs, nanoporous alumina membranes of $25 \mathrm{~nm}$ pore diameter and $50 \mu \mathrm{m}$ thickness (SmartPor membranes; obtained from SmartMembranes $\mathrm{GmBH}$, Halle-Saale) were used as the working electrode. To render the membrane conductive, a $100 \mathrm{~nm}$-thick gold layer was deposited on one side using e-beam evaporation. The Au-capped membranes were placed in contact with each of the electrochemical media at least $15 \mathrm{~min}$ prior to the electrodeposition, in order to ensure a uniform filling of the pores, to establish uniform NW growth. The reference and counter electrodes were an $\mathrm{Ag} / \mathrm{AgCl} / \mathrm{KCl}$ (3 M) electrode and a Pt spiral, respectively. The electrochemical media were de-aerated by argon bubbling before each experiment and maintained under an argon atmosphere during it.

\section{Materials characterization}

After the deposition, the NWs were released from the alumina membrane firstly by removing the Au layer (with a saturated solution of $\mathrm{I}_{2} / \mathrm{I}^{-}$) and subsequently dissolving the alumina membrane in a $1 \mathrm{M} \mathrm{NaOH}$ solution for $30 \mathrm{~min}$ under flashing ultrasound stirring. The released NWs were cleaned with $0.1 \mathrm{M}$ $\mathrm{NaOH}$ solution $(\times 5)$ and Millipore water $(\times 5)$, also under flashing ultrasound stirring.

The morphology of the NWs was examined by using transmission electron microscopy (JEOL 2010F and JEOL 2100). The elemental composition was determined by energy-dispersive $\mathrm{X}$-ray analysis incorporated into a JSM-7100 scanning electron microscope. 
X-ray powder diffraction (XRPD) measurements were performed with a PANalytical X'Pert PRO MPD diffractometer in the Bragg-Brentano reflection $\theta / 2 \theta$ geometry, using $\mathrm{Cu} \mathrm{K}_{\alpha}$ radiation $(\lambda=1.5418 \AA)$. XRPD patterns were obtained after $\theta / 2 \theta$ scans from 20 to $120^{\circ} 2 \theta$, with a step size of $0.017^{\circ} 2 \theta$ and a measuring time of 200 seconds per step and repeating the measurement six times to acquire sufficient statistics. The NW samples (compact and mesoporous) were prepared by deposition of a few drops of a catalyst ink $\left(0.1 \mathrm{mg} \mathrm{mL}^{-1}\right)$ on zero background silicon single crystal sample holders.

Hysteresis loops of the compact and mesoporous NWs (while still embedded in the template) were measured at room temperature using a vibrating sample magnetometer from Micro-Sense, with a maximum external magnetic field of $20 \mathrm{kOe}$, applied either along or perpendicular to the NW axis direction.

In order to determine the electrochemical surface area (ECSA), a catalyst ink was prepared by ultrasonically dispersing $1.0 \mathrm{mg}$ of Pt-based NWs in $2 \mathrm{~mL}$ of ethanol and $8 \mathrm{~mL}$ of Millipore water for $10 \mathrm{~min}$. Subsequently, $3 \mu \mathrm{L}(\times 2)$ of the suspension was pipetted on top of the glassy carbon electrode $\left(0.0314 \mathrm{~cm}^{2}\right)$ and, then, dried under nitrogen flow. This results in a homogeneous coating of NWs, which was used as the working electrode. The ECSA values of the Pt-based NWs were obtained by integrating the charge associated with the adsorption and desorption of hydrogen atoms in cyclic voltammetries recorded in $0.5 \mathrm{M} \mathrm{H}_{2} \mathrm{SO}_{4}$ at $20 \mathrm{mV} \mathrm{s}^{-1}$ at $25{ }^{\circ} \mathrm{C}^{40} \mathrm{An} \mathrm{Ag} / \mathrm{AgCl} / \mathrm{KCl}$ (3 M) $/ \mathrm{H}_{2} \mathrm{SO}_{4}(0.5 \mathrm{M})$ electrode and a Pt spiral were used as the reference and counter electrodes, respectively.

\section{Catalytic activity}

The catalytic activity of the Co-Pt NWs was established by the efficiency of the catalysis of the hydrogenation reaction of 4-nitrophenol. Two different reaction approaches were investigated:

(i) Electroreduction. Chronoamperometric curves were recorded at a constant potential in a $0.7 \mathrm{mM}$ 4-nitrophenol +1 $\mathrm{M} \mathrm{H}_{2} \mathrm{SO}_{4}$ solution in order to promote the electroreduction of 4-nitrophenol in the presence of a working electrode containing the Co-Pt NWs. The electrode was prepared by placing a drop of the NW suspension on the surface of a glassy carbon layer and allowing it to dry. $\mathrm{An} \mathrm{Ag} / \mathrm{AgCl} / \mathrm{KCl}$ (3 M) electrode and a $\mathrm{Pt}$ spiral were used as the reference and counter electrodes, respectively. The 4-nitrophenol was reduced, under magnetic stirring conditions.

(ii) Chemical reduction. Based on the use of an aggressive agent $\left(\mathrm{NaBH}_{4}\right)$ to reduce the 4-nitrophenol in the presence of the Co-Pt NWs (catalysts). For this experiment, $300 \mu \mathrm{L}$ of 4-nitrophenol (0.7 mM solution) was diluted with $1 \mathrm{~mL}$ of Millipore water, followed by the addition of $1 \mathrm{~mL}$ of freshly prepared ice-cold $\mathrm{NaBH}_{4}$ solution $(0.5 \mathrm{M})$, and a given amount of the prepared catalyst ink $\left(0.1 \mathrm{mg} \mathrm{mL}^{-1}\right)$.

Importantly, three different reaction conditions were pursued:

(a) Silent, where no specific actuation was carried out during the reduction reaction. (b) Ultrasound stirring, where NWs were subjected to sonication with a flashing (with $3 / 3$ s ON/OFF cycles) ultrasonic power of $4 \mathrm{~W} \mathrm{~L}^{-1}$ during the reduction reaction.

(c) Magnetic stirring, where NWs were magnetically stirred using a rotary magnetic field (with a maximum applied field of $400 \mathrm{Oe}$ at $20 \mathrm{~Hz}$ - magnetic stirrer RH digital - IKA®) during the reaction time.

All the experiments were conducted at $25{ }^{\circ} \mathrm{C}$.

Note that taking advantage of the well-defined absorption peak of 4-nitrophenol at a wavelength of $400 \mathrm{~nm}$, in both types of reactions the temporal evolution of the catalytic reaction was tracked ex stiu by UV-visible spectroscopy in a quartz cuvette using a UV-1800 Shimadzu UV-visible spectrophotometer.

\section{Results and discussion}

It has been recently shown that magnetic mesoporous NWs with various aspect ratios, with diameters comprised between 100 and $200 \mathrm{~nm}$, can be easily synthesised by means of electrodeposition in the presence of ionic liquid microemulsions. During the synthesis, these microemulsions become confined in the nanochannels of polycarbonate or alumina membranes, acting as a soft-template and defining the NW morphology. ${ }^{39}$ However, the microemulsion approach for electrosynthesising mesoporous NWs of diameters lower than $100 \mathrm{~nm}$ can be rather challenging since, at this length scale, the diameter of the nanochannels becomes commensurate with the hydrodynamic diameter of the ionic liquid droplets in the IL/W microemulsion. Moreover, the higher viscosity of the IL/W microemulsion compared to that of the aqueous solution makes the process even more complex since it tends to hinder the proper penetration of the precursor into the small pores of the membrane.

To grow the narrow mesoporous NWs we follow the synthetic strategy schematically described in Fig. 1, where the porous alumina membrane is dipped in the IL/W microemulsion for at least $15 \mathrm{~min}$ prior to electrodeposition to ensure good diffusion of the IL/W precursor into the narrow holes of the membrane.

Chronoamperometric curves of the synthesis (Fig. 1S $\dagger$ ) show that under stationary conditions the current evolves quickly to quasi-stationary values, which reflects a constant growth regime. The chronoamperometric curves reveal that longer times are necessary to attain the same deposition charge density $\left(2.1 \mathrm{C} \mathrm{cm}^{-2}\right)$ in the case of the microemulsions than in the case of the aqueous electrolytic solutions. Namely, the deposition rate is higher in aqueous solution due to the lower viscosity and relatively higher conductivity of the medium (Table 1). Importantly, the chronoamperometric curves for the IL/W microemulsion case indicate that the microemulsion medium fills the nanochannels of alumina membranes despite its high viscosity, leading to a steady and homogeneous growth, but with a lower stationary current density in comparison with that of the aqueous solution case (with lower viscosity).

As can be seen in Fig. 2, the compact and mesoporous NWs are straight and the diameters are between 23 and $27 \mathrm{~nm}$, which are in the same range as those of the pores of the original alumina membranes (Fig. $2 \mathrm{~S} \dagger$ ). Careful observation of the 


\section{IL/W Microemulsion}
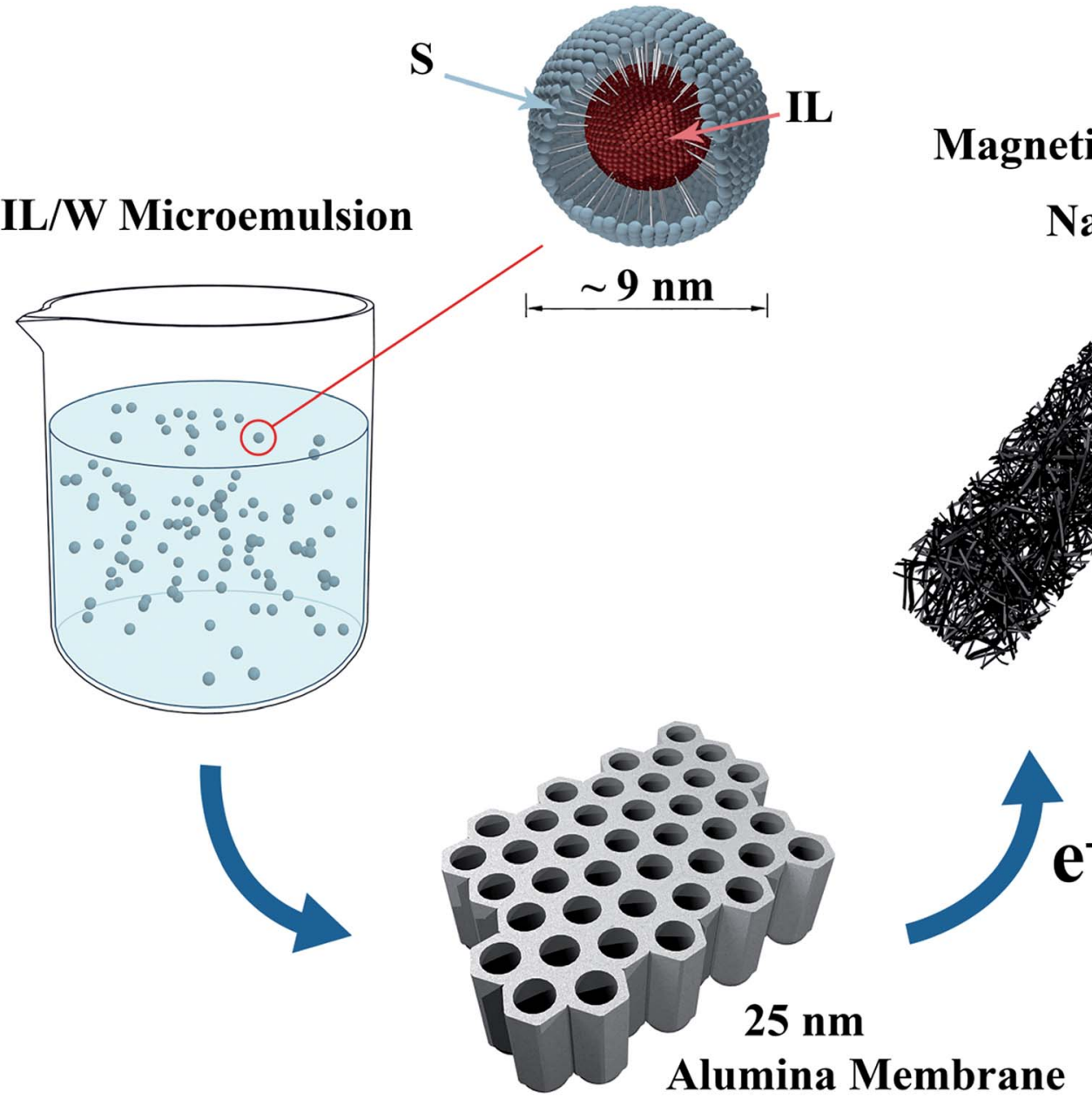

Alumina Membrane

\section{Magnetic Mesoporous} Nanowires
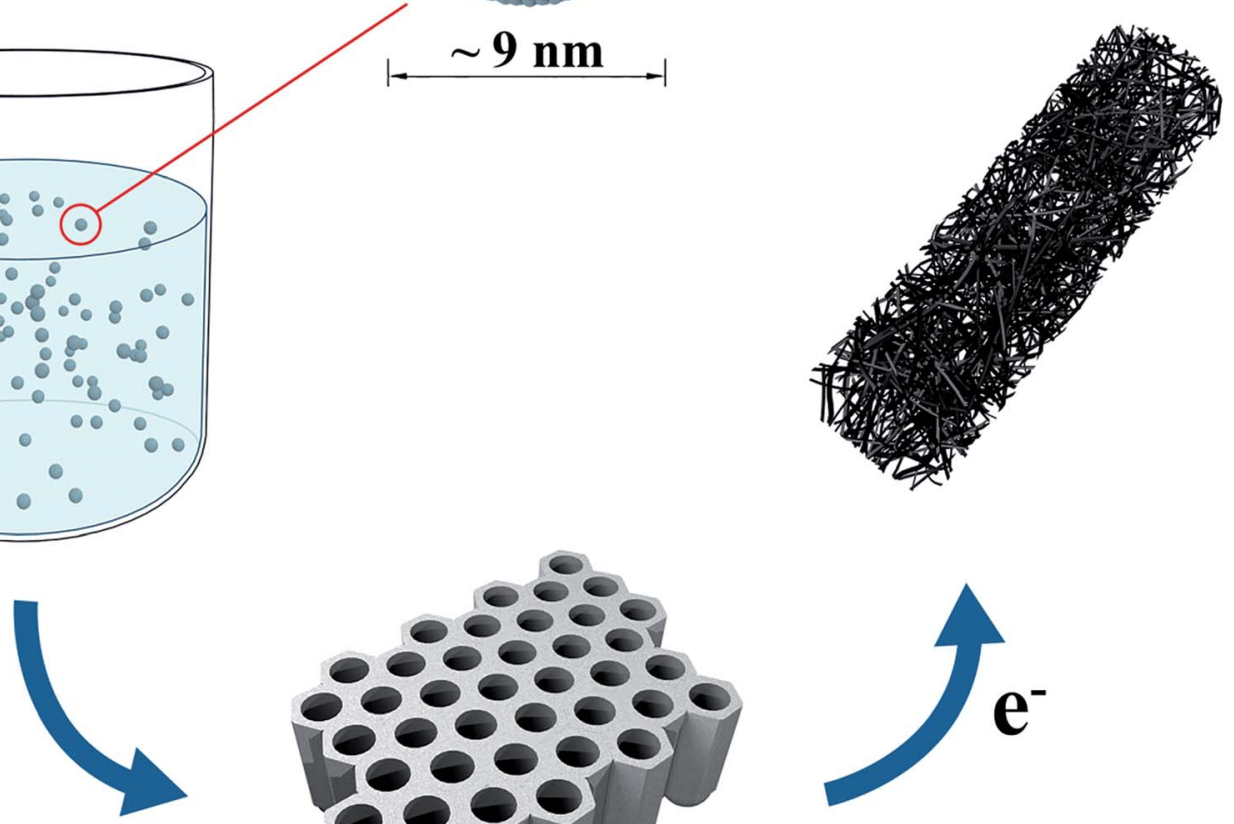

Fig. 1 Schematic representation of the synthesis approach to grow narrow mesoporous NWs.

Table 1 Physicochemical characteristics (conductivity, viscosity and the droplet size) of the electrochemical media used to synthesise the NWs, along with the elemental composition, diameter, deposition rate and ECSA values of the obtained NWs

\begin{tabular}{llllllll}
\hline Media & $\begin{array}{l}\text { Conductivity/ } \\
\mathrm{mS} \mathrm{cm}^{-1}\end{array}$ & $\begin{array}{l}\text { Viscosity/ } \\
\mathrm{mPa} \mathrm{s}\end{array}$ & Droplet size/nm & NW type & $\begin{array}{l}\text { Elemental } \\
\text { composition/at\% }\end{array}$ & $\begin{array}{l}\text { Diameter/ } \\
\mathrm{nm}\end{array}$ & $\begin{array}{l}\text { Deposition } \\
\text { rate/nm s}\end{array}$ \\
\hline W & 11.2 & 0.84 & - & Compact & $\begin{array}{l}\text { ECSA/ } \\
\mathrm{m}^{2} \mathrm{~g}^{-1}\end{array}$ \\
IL/W ME & 6.2 & 34.5 & 9.1 & Mesoporous $/ 29(\mathrm{Pt})$ & $68(\mathrm{Co}) / 32(\mathrm{Pt})$ & $24 \pm 2$ & 5.3 \\
\end{tabular}

mesoporous NWs (Fig. 2b) confirms that there are mesopores homogeneously dispersed all over the entire area of the NWs. The pore size was statistically measured to be approximately $2.6 \pm 0.8 \mathrm{~nm}$. The length of both kinds of NWs can be effectively controlled by adjusting the electrodeposition time, where the growth rates were 5.3 and $1.6 \mathrm{~nm} \mathrm{~s}^{-1}$ for the compact and mesoporous NWs, respectively. Therefore, the IL/W microemulsion approach proves to be a successful strategy to synthesise uniform thin $(\sim 25 \mathrm{~nm})$ mesoporous NWs by electrodeposition. Note that the size of the ionic liquid droplets $(\sim 9 \mathrm{~nm})$ is slightly larger than that of the pores $(\sim 3 \mathrm{~nm})$ observed in the mesoporous NWs (Fig. 2c). This is not surprising as the pores are defined by the size of the ionic liquid droplets, which are actually smaller than the hydrodynamic diameter determined by dynamic light scattering.

Given the importance of the uniform distribution of Co and Pt ions in the stability and catalytic performance, elemental mapping of Co-Pt NWs was performed using TEM-energy dispersive X-ray spectroscopy (TEM-EDS), as shown in Fig. 3Sa and b.† It can be clearly seen that Co and Pt were homogeneously distributed in the entire area of compact and mesoporous NWs, indicating even electrodeposition.

The X-ray diffraction (XRD) patterns of both compact and mesoporous NWs are very similar, indicating the presence of the same crystalline phases (Fig. 4S $\dagger$ ). Quantitative XRD profile analysis (see ESI, Fig. $4 S^{\dagger}$ ) reveals that the NWs are 

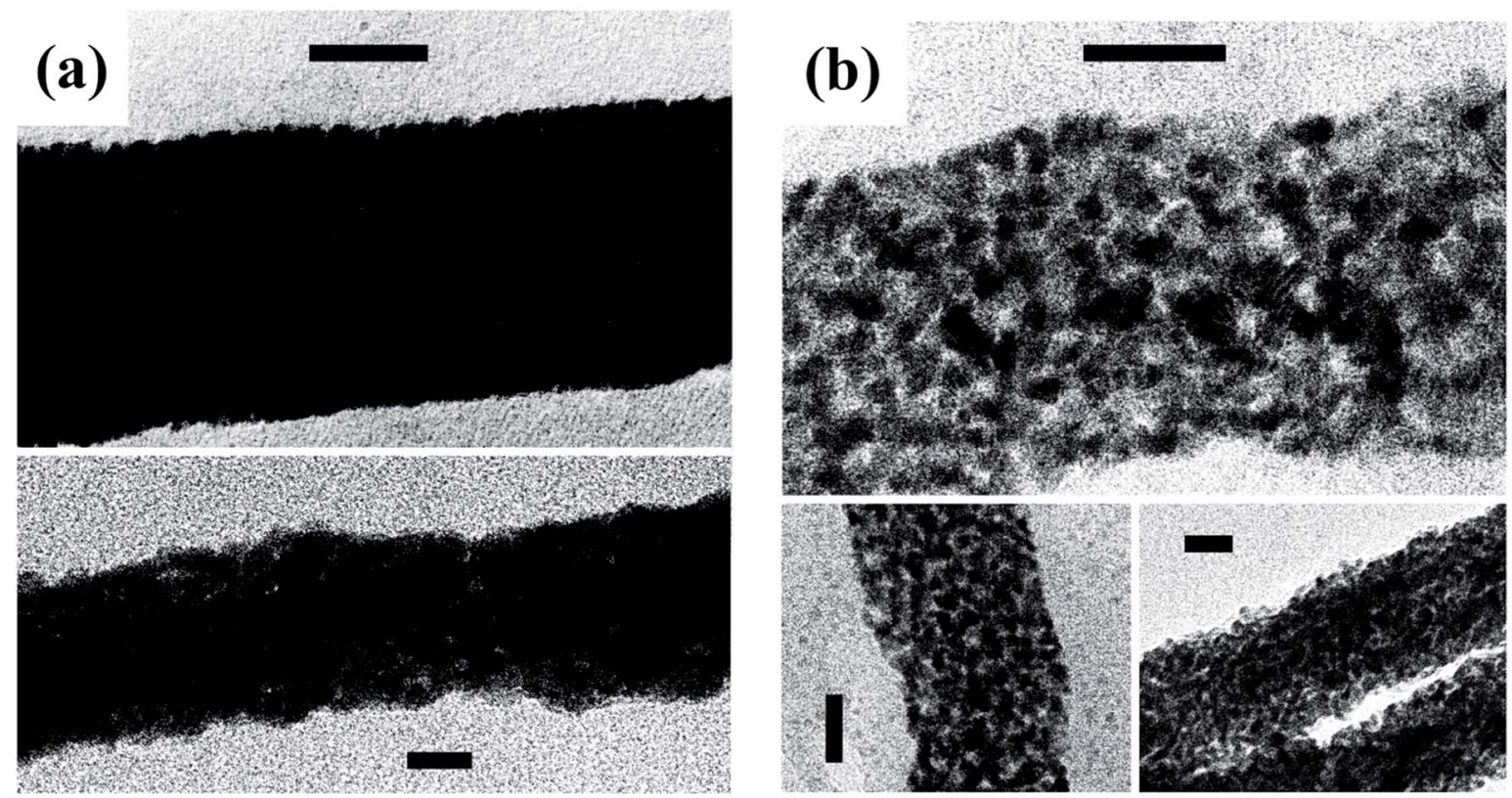

(c)

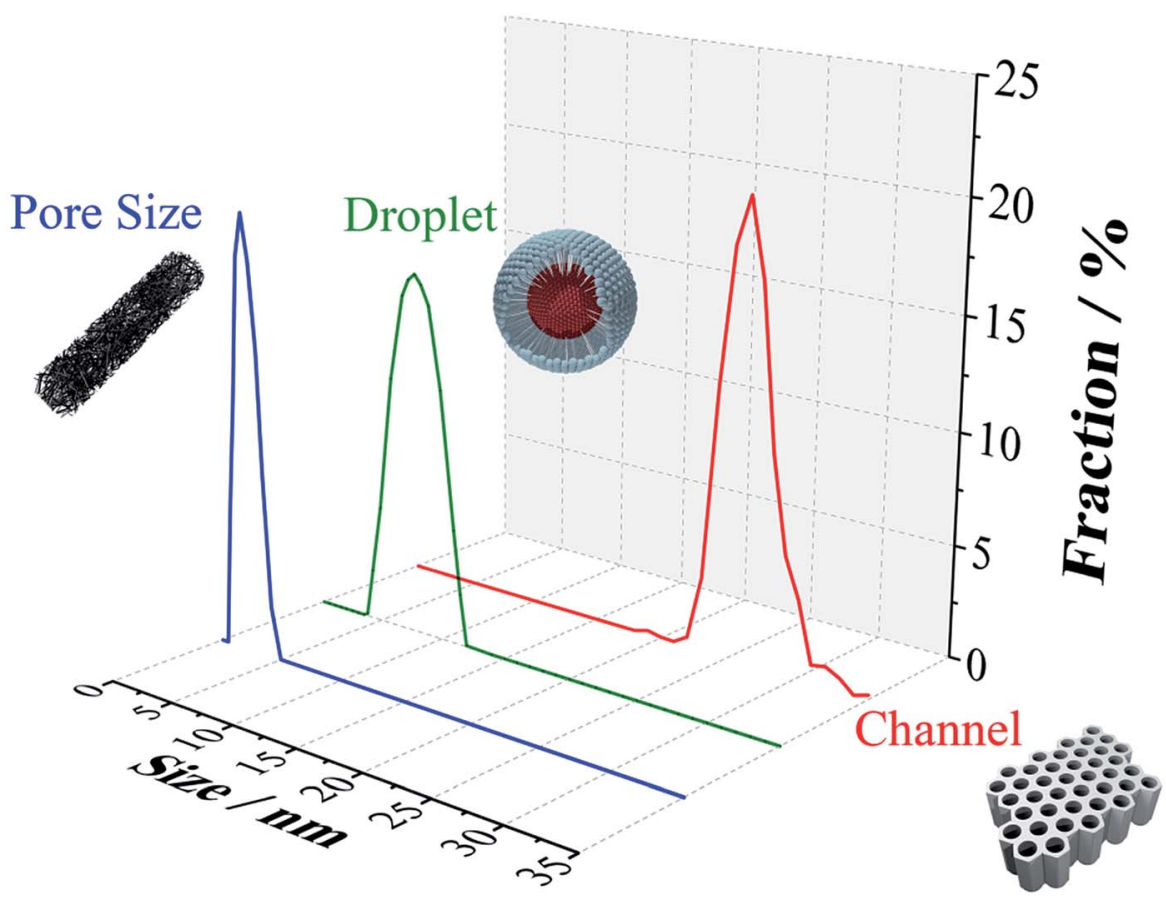

Fig. 2 TEM images of the surface of (a) compact and (b) mesoporous magnetic Co-Pt NWs with diameters of $25 \mathrm{~nm}$. Scale bar: $10 \mathrm{~nm}$. (c) Size distributions of the mesopores, droplets of the ionic liquid and channels of alumina membranes.

composed of two different nanocrystalline phases: (i) fcc $\mathrm{CoPt}_{3}$, with an average crystalline domain size of around 11-12 nm, and (ii) hep CoPt, with a crystalline domain size of about $5 \mathrm{~nm}$. Note that a $\mathrm{CoPt}_{3} \mathrm{fcc}+\mathrm{CoPt}$ hcp phase mixture has also been reported for electrodeposited CoPt films prepared using similar electrolytic baths. Importantly, this implies that the bath determines the crystalline structure of the Co-Pt alloy, even in the interior of narrow pores. ${ }^{41}$ Moreover, the composition of both types of wires (compact and mesoporous) is rather similar, $\sim \mathrm{Co}_{70} \mathrm{Pt}_{30}$ (Table $1 \mathrm{~S} \dagger$ ). Thus, the incorporation of the ionic liquid droplets into the electrolytic bath to form the microemulsion does not modify the crystalline structure or the composition, although the growth rate of the NWs is lower in the microemulsion system. Additionally, the fast Fourier transform of the lattice fringes in the high-resolution TEM (HR-TEM) images (Fig. 3Sc and d $\dagger$ ) confirms the presence of two different phases: $\mathrm{CoPt}_{3} \mathrm{fcc}$ and CoPt hep. 
From the magnetic viewpoint, the fully-dense and mesoporous NWs show semi-hard ferromagnetic properties dominated by shape anisotropy (Fig. $5 \mathrm{~S} \dagger$ ), induced by the large aspect ratio of the wires $(940 \mathrm{~nm} / 25 \mathrm{~nm}=37.6)$. As expected, the magnetic easy axis is along the nanowire axis. The coercivity values along and perpendicular to the nanowire axis are around 1800 Oe and 1400 Oe, respectively, for the compact and mesoporous NWs. Slightly smaller values are obtained in the mesoporous NWs, although the shape anisotropy is still preserved in spite of the existing nanoporosity.

Furthermore, to confirm the high effective area due to the mesoporous structure, we investigated the electrochemical surface areas (ECSAs) of the NWs (Fig. 6S $\dagger$ ). The specific values of the mesoporous NWs (Table 1) show a reasonably large surface area $\left(331 \mathrm{~m}^{2} \mathrm{~g}^{-1}\right)$ with respect to that of the compact ones $\left(86 \mathrm{~m}^{2} \mathrm{~g}^{-1}\right)$.

The reduction of 4-nitrophenol is first attempted by electrocatalysis. This approach avoids the use of aggressive reducing agents such as sodium borohydride. However, the electrochemical reduction of nitro-aromatic compounds is complicated as a consequence of the various chemical transformations involved in the process, which are strongly dependent on $\mathrm{pH}$, solvent and catalytic nature. ${ }^{\mathbf{4 2 - 4 5}}$ Nonetheless, in protic media, the predominant reduction product is either aminophenol and/or aniline, depending upon the strength of the acidic medium, ${ }^{\mathbf{4 6}-48}$ while in aprotic media it is 4-hydroxylaminophenol. For example, in an acidic electrolyte $\left(10 \% \mathrm{H}_{2} \mathrm{SO}_{4}\right)$, nitrophenol is reduced to aminophenol in a virtually quantitative yield. ${ }^{49,50}$ Therefore, based on the previously reported results from the literature, a potential of $-0.35 \mathrm{~V} v s$. $\mathrm{Ag} / \mathrm{AgCl} / \mathrm{KCl}(3 \mathrm{M}) / \mathrm{H}_{2} \mathrm{SO}_{4}\left(\begin{array}{ll}0.5 & \mathrm{M}\end{array}\right)$ was selected to explore the electroreduction of 4-nitrophenol in acidic media (1 M $\left.\mathrm{H}_{2} \mathrm{SO}_{4}\right){ }^{51,52}$ Therefore, in order to use Co-Pt NWs for an effective electrochemically catalysed hydrogenation of 4-nitrophenol, it is important to evaluate their stability, as well as their catalytic activity and chemoselectivity in this aggressive environment. Moreover, for comparison, pure Pt and Co mesoporous NWs were also employed. (a)

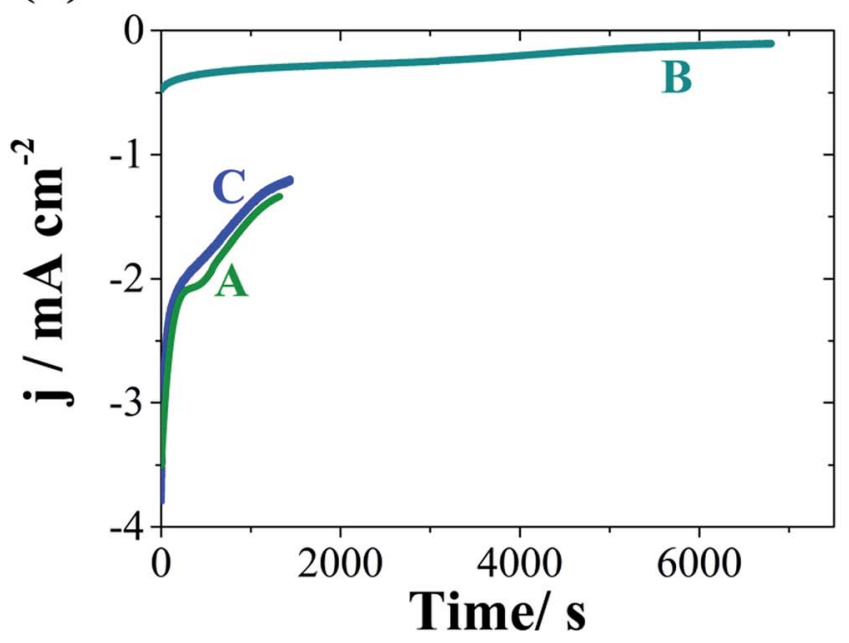

(c)

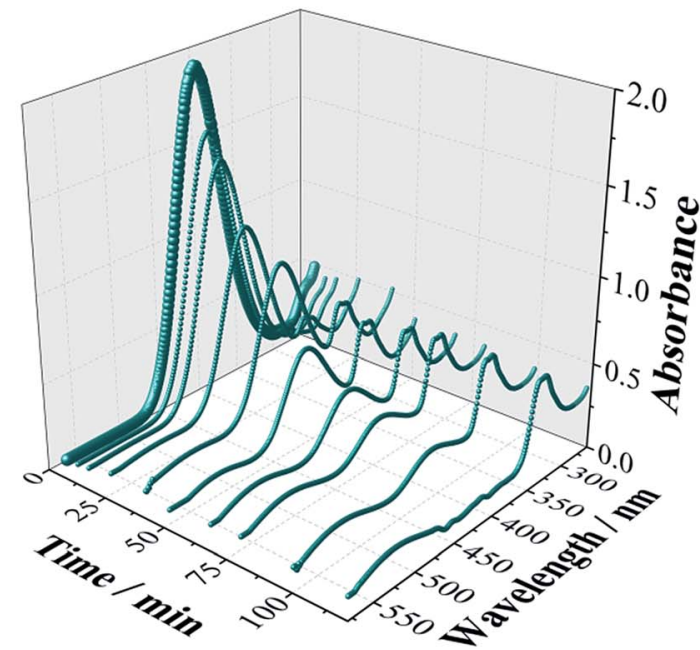

(b)

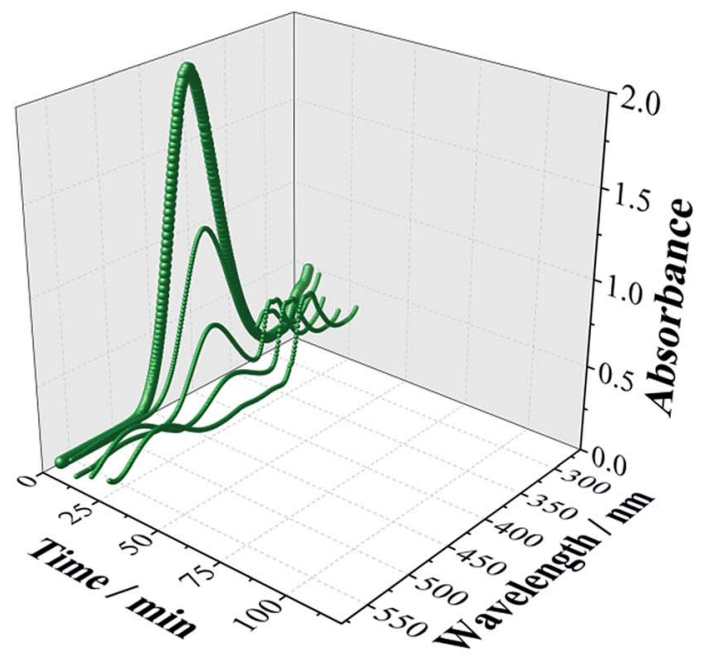

(d)

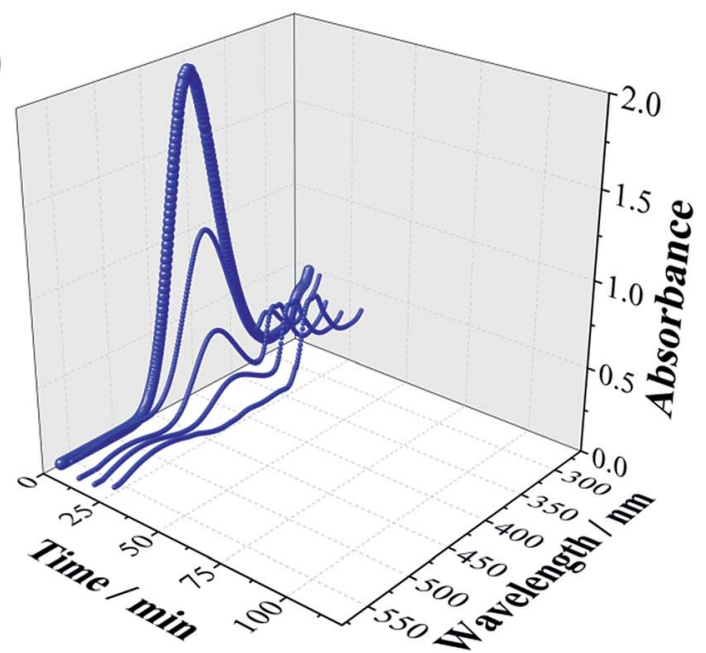

Fig. 3 (a) Chronoamperometric curves (recorded at $-0.35 \mathrm{~V}$ vs. Ag/AgCl/KCl (3 M)/ $\mathrm{H}_{2} \mathrm{SO}_{4}(0.5 \mathrm{M})$ ) of (A) Pt mesoporous, (B) Co-Pt compact and (C) Co-Pt mesoporous NWs for the electroreduction of 4-nitrophenol. Time-dependent UV-visible spectra of 4-NP electrocatalysed reduction using (b) Pt mesoporous, (c) Co-Pt compact and (d) Co-Pt mesoporous NWs. 
Fig. 3a shows the chronoamperometric curves of the electroreduction of 4-nitrophenol using compact or mesoporous NWs. It can be seen that a significantly higher current density is registered when mesoporous NWs are used as electrocatalysts due to the high active surface area. For this experiment, $300 \mu \mathrm{L}$ of the reactant solution was basified with $\mathrm{KOH} \mathrm{(5} \mathrm{M)} \mathrm{and} \mathrm{diluted}$ to $2 \mathrm{~mL}$ before registering the UV-visible spectra. The reaction kinetics can be easily monitored from the time-dependent absorption spectra (Fig. 3b-d), which shows the expected profile (Fig. 7S $\dagger$ ), in which the absorption peak at $400 \mathrm{~nm}$ ascribed to nitro-compounds decreases, while the 4-aminophenol peak at $304 \mathrm{~nm}$ concomitantly develops. This is accompanied by a change in the colour of the solution from yellowish to colourless. As shown in Fig. 3a, no signal could be detected when Co mesoporous NWs were used. This is expected since a sulphuric acid medium attacks and dissolves these Co nanostructures. Consequently, pure Co mesoporous NWs could not be used as electrocatalysts in an acidic environment. As shown in Table 2, the required time for the total reduction (i.e., no detection of 4-nitrophenol in the UV-visible spectra) is rather short for both the Pt and Co-Pt catalysts. However, during the electrocatalysis, two more peaks can be identified at $317 \mathrm{~nm}$ (a considerably large peak) and $504 \mathrm{~nm}$ (a weak peak), indicating the formation of other by-products. As shown in Fig. 3b the use of mesoporous Pt NWs delivers high catalytic activity, but rather poor chemoselectivity as the peak at $504 \mathrm{~nm}$ is significantly larger than that obtained when Co-Pt NWs were used as electrocatalysts. Therefore, the use of Pt-based alloys can maintain catalytic activity, while increasing chemoselectivity. Note that Pt-group metals are well known to allow high catalytic activity although with relatively poor selectivity, whereas transition metal-based catalysts deliver high chemoselective hydrogenation of nitro-aromatic compounds.

Therefore, Co-Pt compact and mesoporous catalysts exhibited excellent electrocatalytic performance with high catalytic activity for the electrocatalytic reduction of 4-nitrophenol and relatively moderate selectivity to 4-nitrophenol. Importantly, the mesoporous morphology shows an almost 5-fold improvement in the reduction times as compared to that of the compact ones, as expected from the increased surface area. Moreover, in order to demonstrate the stability, recyclability and durability of the NWs, even in the acidic environment, the cyclic voltammetries

Table 2 Comparison of the potential, reduction time, ECSA values and elemental composition before and after used compact and mesoporous Co-Pt NWs and pure Co and Pt mesoporous NWs for the electroreduction of 4-NP

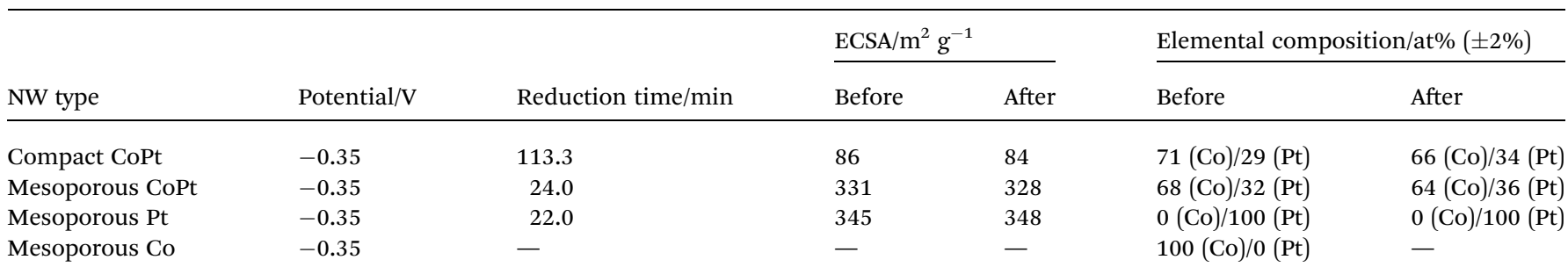

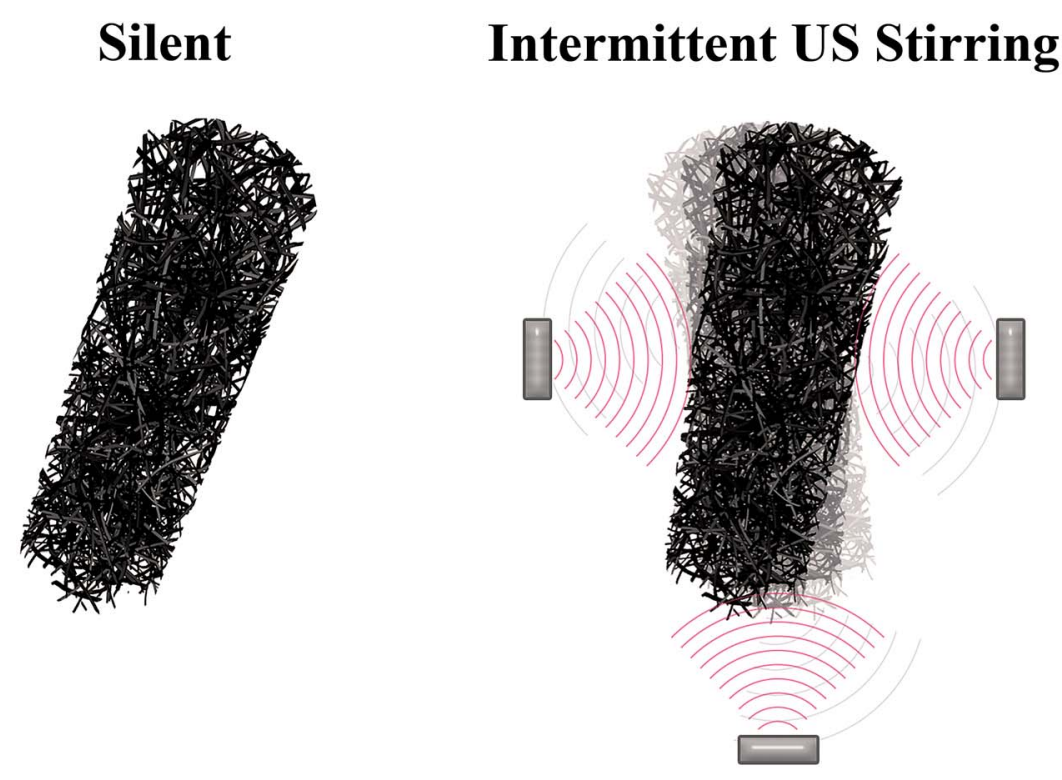

\section{Magnetic Stirring}

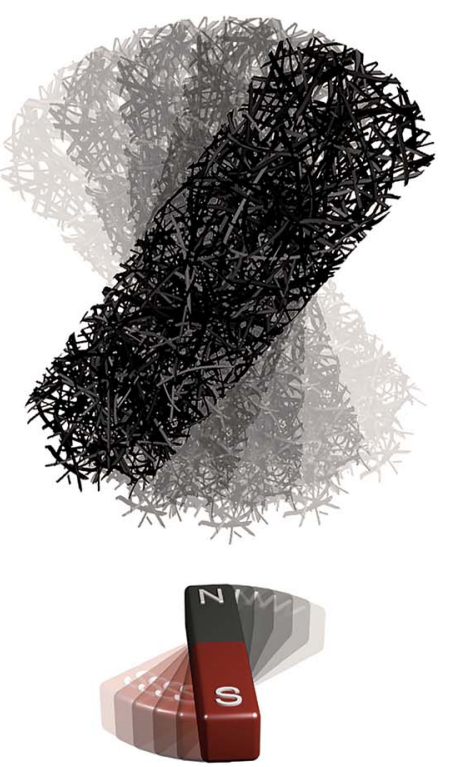

Fig. 4 Schematic representation of various conditions to catalyse the 4-NP reduction. 
(a)

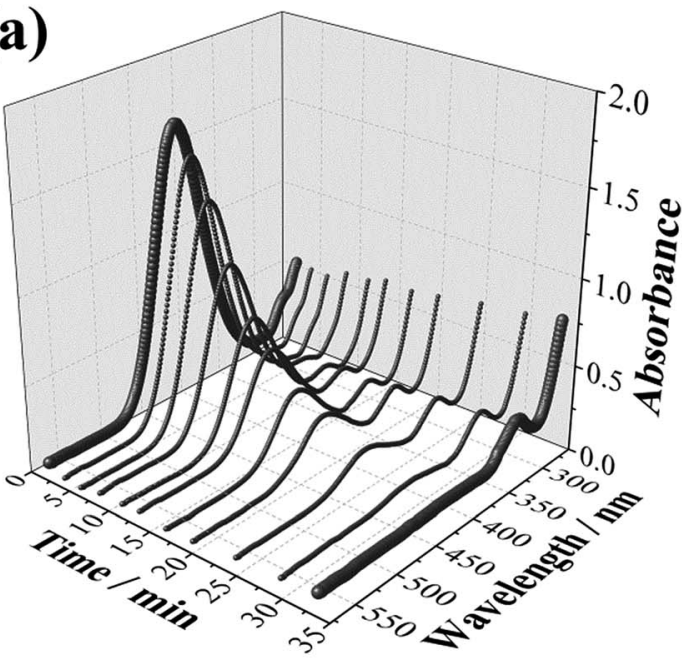

(c)

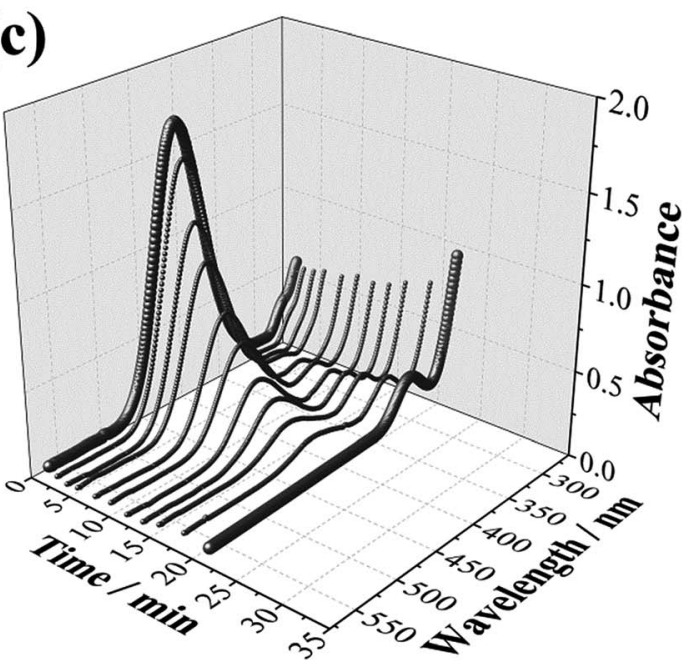

(e)

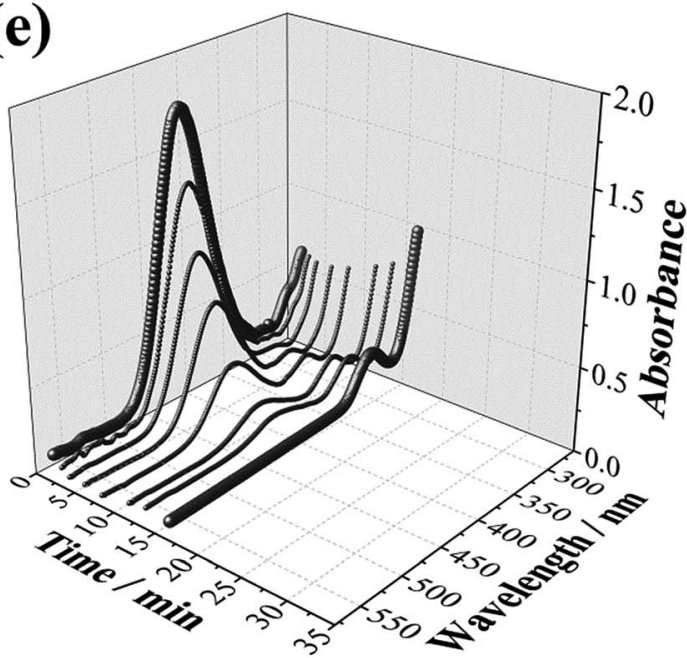

(b)

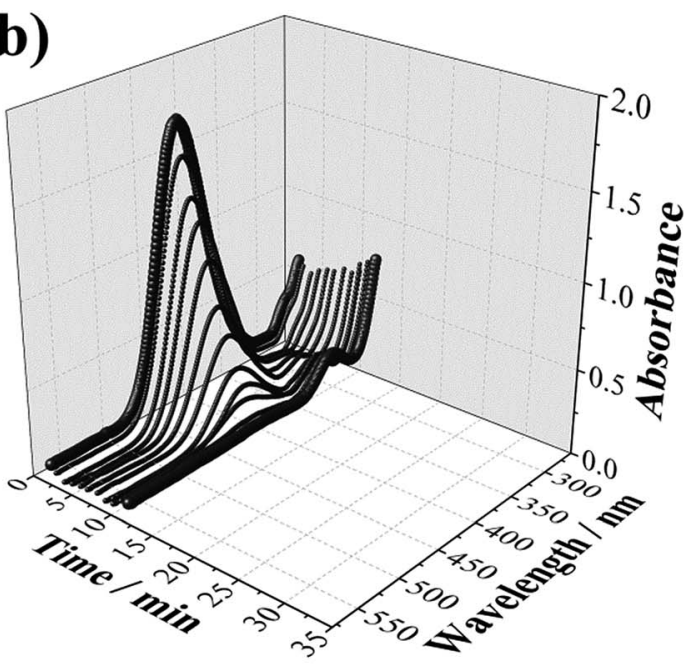

(d)

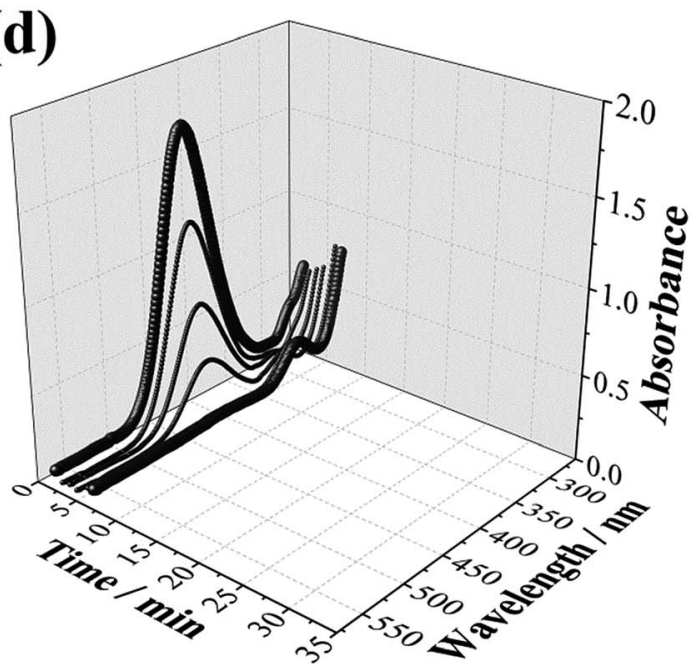

(f)

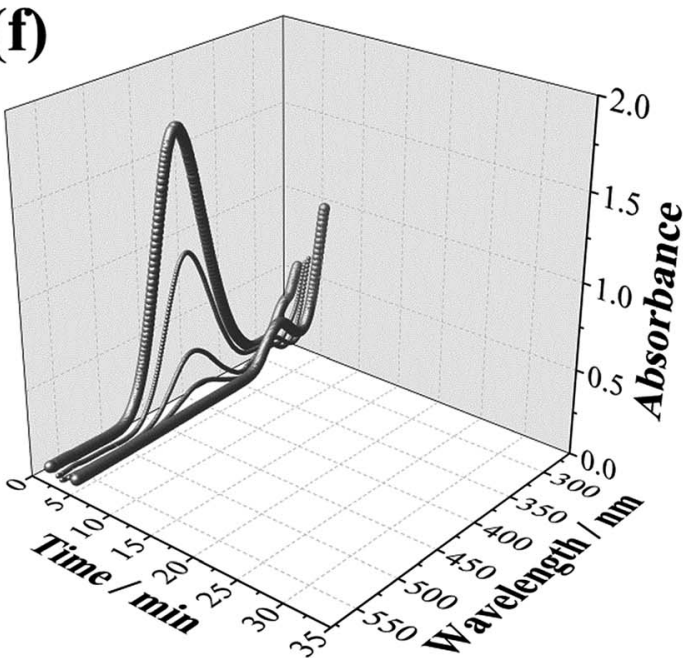

Fig. 5 Time-dependent UV-visible spectra of 4-NP catalysed reduction under silent (panels a and b, for compact and mesoporous NWs, respectively), intermittent ultrasound stirring (panels $\mathrm{c}$ and $\mathrm{d}$, for compact and mesoporous NWs, respectively) and magnetic stirring (panels $\mathrm{e}$ and $\mathrm{f}$, for compact and mesoporous NWs, respectively) conditions, in which $12 \mu \mathrm{L}$ of a suspension of the catalyst $\left(0.1 \mathrm{mg} \mathrm{mL}{ }^{-1}\right) \mathrm{were}^{\mathrm{added}}$.

(CVs), and elemental analysis of the NWs after being used as electrocatalysts, were also registered, since cobalt or cobalt oxides could be dissolved. However, non-significant changes in
ECSA (Fig. 8S†ं) values are observed (Table 2). As could be seen, the Co content of the NWs decreases slightly as a result of the dealloying (i.e., leaching of the surface Co atoms) in this acidic 
environment. However, these variations are not significant, since after the $6^{\text {th }}$-six set of cycles, the Co content was only reduced by $18 \%$ and $7 \%$ for compact and mesoporous NWs, respectively. Nevertheless, dealloying could be a limiting factor for Co-Pt NWs after a large number of uses. Thus, given the fast reduction time and the excellent recyclability, mesoporous NWs present a viable catalytic material for the hydrogenation of 4-nitrophenol by electrocatalysis. Importantly, this approach, despite the moderate selectivity, can be considered more environmentally friendly than conventional catalysis since it avoids the use of the strong reducing agent $\mathrm{NaBH}_{4}$.

The catalytic performance of NWs is also investigated by standard catalysis using $\mathrm{NaBH}_{4}$, where three different reaction conditions are investigated: silent, intermittent ultrasound and magnetic stirring $(20 \mathrm{~Hz})$ (see Fig. 4). Therefore, the use of compact or mesoporous and magnetic or non-magnetic nanostructures allow us to establish the influence of surface area and magnetic actuation on the hydrogenation of 4-nitrophenol. In all cases, $12 \mu \mathrm{L}$ of a NW suspension $\left(0.1 \mathrm{mg} \mathrm{mL}^{-1}\right)$ were added to the reactant solution. As can be seen in Fig. 5 and $9 \mathrm{~S}, \dagger$ the intensity of the peak in the UV-Vis corresponding to the nitrocompound tends to decrease with time until it is no longer observed, revealing the end of the reduction process. This occurs at different times depending on the material type and experimental conditions. Concurrently, as the peak for 4-nitrophenol decreases, a peak at $304 \mathrm{~nm}$ (corresponding to 4-aminophenol) appears. However, in contrast to the electrocatalysis case, here no other by-products are detected, thus evidencing the high selectivity of this approach. Remarkably, the mesoporous NWs exhibit considerably shorter reduction times than those of the compact ones in all three conditions. In particular, the Co-Pt mesoporous NWs under magnetic stirring conditions present exceptional performance.

Since in this catalytic reaction the kinetics can be considered pseudo-first-order with respect to 4-nitrophenol owing to the great excess of $\mathrm{NaBH}_{4}$ concentration, the reaction rate is roughly independent of the $\mathrm{NaBH}_{4}$ concentration. This allows obtaining the kinetic rate pseudo-constants of the different conditions. Fig. 6 and 10S $\uparrow$ display the logarithmic plot of the absorbance $\left(-\ln \left(A / A_{0}\right)\right)$ as a function of the reaction time, from which the apparent kinetic rate constant $\left(k_{\text {app }}\right)$ can be calculated. The apparent kinetic rate constant is not proportional to the total surface area of the NWs, as the bimetallic character and porosity provide synergetic effects to the catalytic reduction. Meanwhile, the normalized rate constant $\left(k_{\text {nor }}\right)$ is also determined, which is associated with the amount of the catalyst $\left(k_{\text {nor }}=k_{\text {app }} / m_{\text {catalyst }}\right)$. As listed in Table 3 , the $k_{\text {app }}$ value of mesoporous Co-Pt NWs is $5.7 \mathrm{~ms}^{-1}$, which is about 3 times
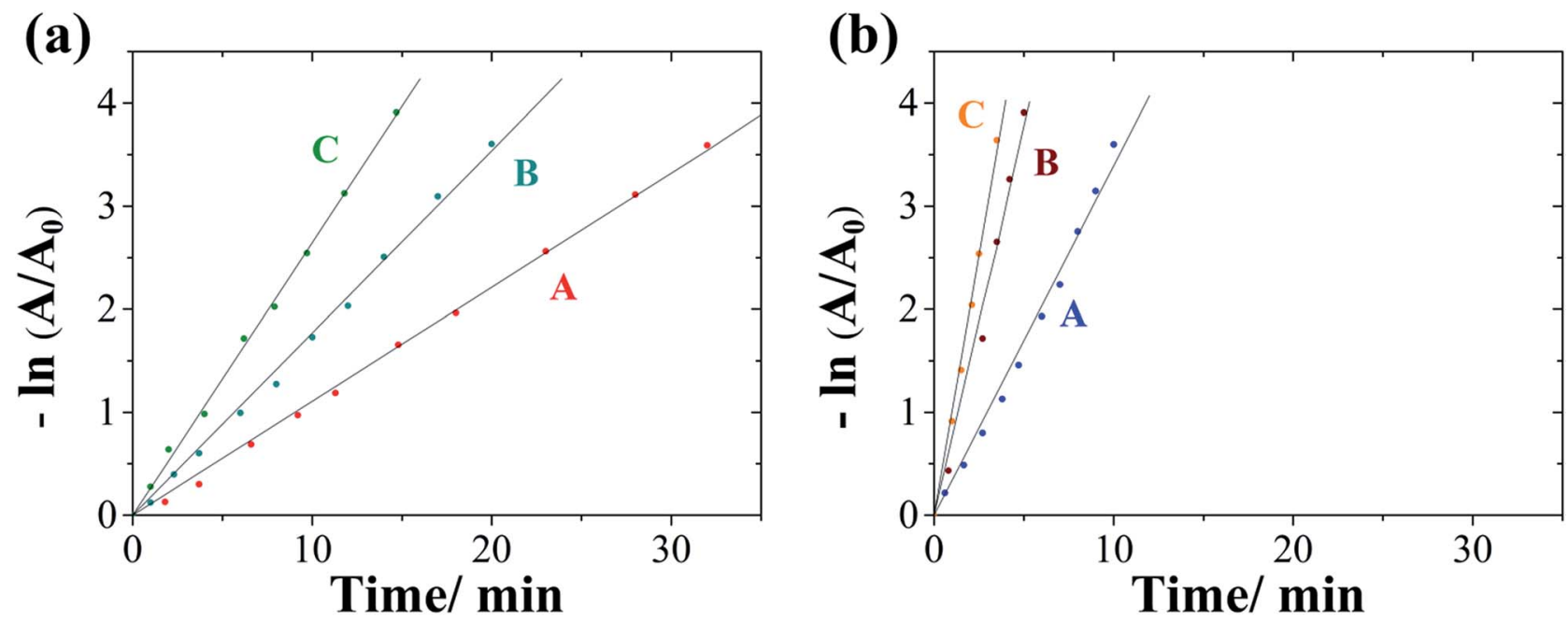

Fig. 6 Plots of $-\ln \left(A / A_{0}\right)$ against reaction time for the catalysed reduction of 4-NP by (a) compact and (b) mesoporous NWs under silent (A), intermittent ultrasound stirring $(B)$ and magnetic stirring $(C)$ conditions.

Table 3 Comparison of the catalytic performance of compact and mesoporous NWs for the reduction of 4-nitrophenol under identical silent, intermittent ultrasound and magnetic stirring conditions

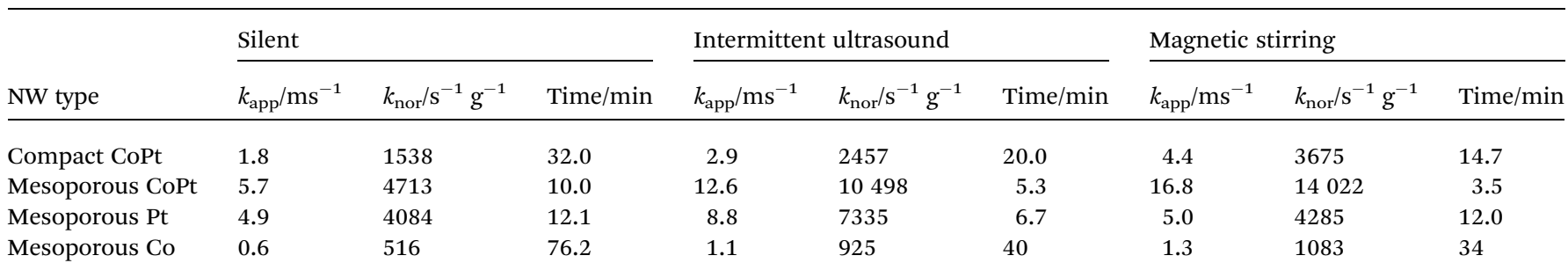


higher than that of the compact ones $\left(1.8 \mathrm{~ms}^{-1}\right)$. Remarkably, the normalized rate constant under silent conditions for $\mathrm{Co}-\mathrm{Pt}$ mesoporous NWs is slightly higher than that of the pure Pt mesoporous NWs and significantly higher than that of pure Co mesoporous NWs. This improved catalytic activity could be attributed to the surface structure (bifunctional nature of the catalyst surface, reduction of Pt-Pt distances, and suppression in d-band centres, amongst others) and the synergetic effects of Co and Pt. ${ }^{53-56}$ In addition, intermittent ultrasound enhances the catalytic performance in all cases as a consequence of the mass transport improvement. On the other hand, magnetic actuation considerably improves the performance only in the case of magnetic catalysts, Co and Co-Pt, whereas for the nonmagnetic Pt catalyst the change is negligible. This indicates that magnetic actuation improves catalytic activity as a result of the movement of the nanowires in the rotating magnetic field, which facilitates the contact between the reagent and the catalyst surface. Therefore, the application of a magnetic field does not necessarily promote the kinetics of this catalysed reaction, but greatly improves the mass flow.

In addition, when comparing $k_{\text {nor }}$ of the mesoporous NWs under magnetic stirring conditions with state-of-the-art catalysts for the 4-nitrophenol reduction reaction (Table $2 \mathrm{~S}_{\dagger}^{\dagger}$ ), it is evident that the Co-Pt mesoporous NWs clearly outperform the other catalysts with more than 8 times better $k_{\text {nor }}$ than that of the next-best catalyst. ${ }^{57-64}$ Moreover, the fact that the mesoporous NWs are Co-rich implies that they are considerably more inexpensive than many of the other proposed catalysts, usually based on expensive noble metals, like Pt, Ag or Au.

Finally, it is worth emphasizing that both, compact and mesoporous NWs, exhibit excellent recyclability without significant reduction in the conversion even after eight cycles (Fig. 11S $\dagger$ ), in which the same stiff limit for each catalyst and conditions are maintained for completion of the reaction. These results clearly prove that CoPt mesoporous NWs under magnetic stirring conditions are extremely efficient catalysts for the 4-nitrophenol reduction. Moreover, the elemental composition and electrochemical surface area of Co-Pt NWs after eight cycles (Table 4) were essentially constant, which, combined with the virtually unchanged catalytic activity, demonstrate their excellent stability. This combined with their cost-effectiveness and their excellent recyclability make Co-Pt mesoporous NWs a very viable option for their commercial exploitation in wastewater cleaning compared with other catalysts.

Table 4 Comparison of the ECSA values and elemental composition before and after used compact and mesoporous Co-Pt NWs and pure Co and Pt mesoporous NWs for the hydrogenation of 4-NP

\begin{tabular}{llllll}
\hline & \multicolumn{3}{c}{ ECSA $/ \mathrm{m}^{2} \mathrm{~g}^{-1}$} & & \multicolumn{2}{l}{$\begin{array}{l}\text { Elemental composition/at\% } \\
( \pm 2 \%)\end{array}$} \\
\cline { 2 - 3 } \cline { 6 - 7 } NW type & Before & After & & Before & After \\
\hline Compact CoPt & 86 & 89 & & $71(\mathrm{Co}) / 29(\mathrm{Pt})$ & $68(\mathrm{Co}) / 32(\mathrm{Pt})$ \\
Mesoporous CoPt & 331 & 330 & & $68(\mathrm{Co}) / 32(\mathrm{Pt})$ & $70(\mathrm{Co}) / 30(\mathrm{Pt})$ \\
Mesoporous Pt & 345 & 350 & & $0(\mathrm{Co}) / 100(\mathrm{Pt})$ & $0(\mathrm{Co}) / 100(\mathrm{Pt})$ \\
Mesoporous Co & - & - & & $100(\mathrm{Co}) / 0(\mathrm{Pt})$ & $100(\mathrm{Co}) / 0(\mathrm{Pt})$
\end{tabular}

\section{Conclusions}

Summarizing, we have shown that IL/W microemulsions in alumina templates can be used as an efficient way to synthesize uniform mesoporous magnetic Pt, Co and Co-Pt NWs with diameters as small as $25 \mathrm{~nm}$, despite the high viscosity of the microemulsion and the commensurability between the template pores and the ionic liquid droplets. Moreover, the Co-Pt mesoporous NWs have been demonstrated to be excellent catalysts for the reduction of highly toxic 4-nitrophenol both by electrocatalysis and by conventional catalysis (using $\mathrm{NaBH}_{4}$ ). In the case of electrocatalysis, despite the mesoporous NWs showing only moderate selectivity, the process can be considered as more environmentally friendly since it avoids the use of $\mathrm{NaBH}_{4}$. On the other hand, the catalytic activity of the mesoporous NWs when used together with $\mathrm{NaBH}_{4}$ depends markedly on the reaction conditions (silent, ultrasound and magnetic stirring). Actually, the performance of the magnetically actuated mesoporous NWs is far better than the existing catalysts for the reduction of 4-nitrophenol. Moreover, given their magnetic character, these mesoporous NWs are readily recyclable without the loss of catalytic activity for both types of reduction processes. The Co-Pt mesoporous NWs have been demonstrated to be very powerful catalysts (outperforming even pure Pt mesoporous NWs) not only due to their very high active surface area, but also as a consequence of their alloyed character. Namely, the presence of cobalt in the alloy (i) confers the NWs with a magnetic character, allowing magnetic actuation, (ii) enhances the catalytic activity and chemoselectivity and (iii) implies significant economical savings with respect to the use of pure Pt catalysts. These results pave the way to develop highly efficient catalysts to commercialize new viable wastewater treatment approaches.

\section{Acknowledgements}

This work was supported by the EU ERDF (FEDER) fund and the Spanish Government grants TEC2014-51940-C2-2-R and MAT2014-57960-C3-1-R from Ministerio de Economía y Competitividad (MINECO) - the latter co-financed by FEDER. Partial funding from the European Research Council (Consolidator Grant, project n. 648454, SPIN-PORICS) and the Generalitat de Catalunya (2014-SGR-1015 project) is acknowledged. The authors thank the CCiT-UB for the use of their equipment. ICN2 acknowledges support from the Severo Ochoa Program (MINECO, Grant SEV-2013-0295).

\section{Notes and references}

1 P. Kovacic and R. Somanathan, J. Appl. Toxicol., 2014, 34, 810.

2 A. Niaz, J. Fischer, J. Barek, B. Yosypchuk, Sirajuddin and M. I. Bhanger, Electroanalysis, 2009, 21, 1786.

3 P. Mulchandani, C. H. Hangarter, Y. Lei, W. Chen and A. Mulchandani, Biosens. Bioelectron., 2005, 21, 523.

4 K.-S. Ju and R. E. Parales, Microbiol. Mol. Biol. Rev., 2010, 74, 250. 
5 J. Feng, L. Su, Y. Ma, C. Ren, Q. Guo and X. Chen, Chem. Eng. J., 2013, 221, 16.

6 J. Li, D. Kuang, Y. Feng, F. Zhang, Z. Xu and M. Liu, J. Hazard. Mater., 2012, 201-202, 250.

7 N. Modirshahla, M. A. Behnajady and S. MohammadiAghdam, J. Hazard. Mater., 2008, 154, 778.

8 M. S. Dieckmann and K. A. Gray, Water Res., 1996, 30, 1169.

9 S. Yuan, M. Tian, Y. Cui, L. Lin and X. Lu, J. Hazard. Mater., 2006, 137, 573.

10 T. R. Mandlimath and B. Gopal, J. Mol. Catal. A: Chem., 2011, 350, 9.

11 J.-J. Lv, A.-J. Wang, X. Ma, R.-Y. Xiang, J.-R. Chen and J.-J. Feng, J. Mater. Chem. A, 2015, 3, 290.

12 P. Deka, R. C. Deka and P. Bharali, New J. Chem., 2014, 38, 1789.

13 J. Li, C.-Y. Liu and Y. Liu, J. Mater. Chem., 2012, 22, 8426.

14 A. T. Massenova and L. R. Sassykova, Eurasian Chem.Technol. J., 2000, 2, 101.

15 K. Junge, B. Wendt, N. Shaikh and M. Beller, Chem. Commun., 2010, 46, 1769.

16 S. Zhang, C.-R. Chang, Z.-Q. Huang, J. Li, Z. Wu, Y. Ma, Z. Zhang, Y. Wang and Y. Qu, J. Am. Chem. Soc., 2016, 138, 2629.

17 Opinion on para-Aminophenol, Adopted by the Scientific Committee on Consumer Products of European Commission, March 2005.

18 Ö. Sahin, N. Genli and M. Özdemir, Chem. Eng. Process., 2005, 44, 1.

19 T. Komatsu and T. Hirose, Appl. Catal., A, 2004, 276, 95.

20 R. Nie, J. Wang, L. Wang, Y. Qin, P. Chen and Z. Hou, Carbon, 2012, 50, 586.

21 S. Kataoka, Y. Takeuchi, A. Harada, T. Takagi, Y. Takenaka, N. Fukaya, H. Yasuda, T. Ohmori and A. Endo, Appl. Catal., A, 2012, 427, 119.

22 J. Gu, Z. Zhang, P. Hu, L. Ding, N. Xue, L. Peng, X. Guo, M. Lin and W. Ding, ACS Catal., 2015, 5, 6893.

23 R. V. Jagadeesh, A. E. Surkus, H. Junge, M.-M. Pohl, J. Radnik, J. Rabeah, H. Huan, V. Schunemann, A. Bruckner and M. Beller, Science, 2013, 342, 1073.

24 R. V. Jagadeesh, D. Banerjee, P. B. Arockiam, H. Junge, K. Junge, M.-M. Pohl, J. Radnik, A. Bruckner and M. Beller, Green Chem., 2015, 17, 898.

25 R. V. Jagadeesh, T. Stemmler, A.-E. Surkus, H. Junge, K. Junge and M. Beller, Nat. Protoc., 2015, 10, 548.

26 M. Cortés, A. Serrà, E. Gómez and E. Vallés, Electrochim. Acta, 2011, 56, 8232 .

27 A. Serrà, E. Gómez, J. F. López-Barbera, J. Nogués and E. Vallés, ACS Nano, 2014, 8, 4630.

28 Y. Wang and H. Yang, J. Am. Chem. Soc., 2005, 127, 5316.

29 E. S. Toberer, T. D. Schladt and R. Seshadri, J. Am. Chem. Soc., 2006, 128, 1462.

30 Z. C. Bai, B. Sun, N. Fan, Z. C. Ju, M. H. Li, Q. Xu and Y. T. Qian, Chem.-Eur. J., 2012, 18, 5319.

31 Y. Ren, Z. Ma, R. E. Morris, Z. Liu, F. Jiao, S. Dai and P. G. Bruce, Nat. Commun., 2013, 4, 2015.

32 C. Li, T. Sato and Y. Yamauchi, Angew. Chem., Int. Ed., 2013, 52,8050 .
33 Y. Yamauchi, A. Tonegawa, M. Komatsu, H. Wang, L. Wang, Y. Nemoto, N. Suzuki and K. Kuroda, J. Am. Chem. Soc., 2012, 134, 5100.

34 X. Zhang, W. Lu, J. Dai, L. Bourgeois, N. Hao, H. Wang, D. Zhao and P. A. Webley, Angew. Chem., 2010, 122, 10299.

35 A. Serrà, E. Gómez and E. Vallés, Electrochim. Acta, 2015, 174, 630.

36 A. Serrà, E. Gómez and E. Vallés, Int. J. Hydrogen Energy, 2015, 40, 8062.

37 A. Serrà, E. Gómez, I. V. Golosovsky, J. Nogués and E. Vallés, J. Mater. Chem. A, 2016, 4, 7805.

38 A. Serrà, E. Gómez, M. Montiel and E. Vallés, RSC Adv., 2016, 6, 47931.

39 Y. Gao, S. Han, B. Han, G. Li, D. Shen, Z. Li, J. Du, W. Hou and G. Zhang, Langmuir, 2005, 21, 568.

40 N. Tian, Z.-Y. Zhou, S.-G. Sun, Y. Ding and L. W. Zhong, Science, 2007, 316, 732.

41 S. Grau, M. Montiel, E. Gómez and E. Vallés, Electrochim. Acta, 2013, 109, 187.

42 A. Pelekourtsa, N. Missaelidis and D. Jannakoudakis, Chim. Chron., 1997, 26, 39.

43 P. Zuman and Z. Fijalek, J. Electroanal. Chem., 1990, 296, 583. 44 C. L. Forryan and R. G. Compton, Phys. Chem. Chem. Phys., 2003, 5, 4226.

45 D. S. Silvester, A. J. Wain, L. Aldous, C. Hardacre and R. G. Compton, J. Electroanal. Chem., 2006, 596, 131.

46 J. Marquez and D. J. Pletcher, J. Appl. Electrochem., 1980, 10, 567.

47 Y. Sun and W. Xu, Electrochim. Acta, 1993, 38, 1753.

48 A. Cyr, P. Huot, G. Belot and J. Lessard, Electrochim. Acta, 1990, 35, 147.

49 N. M. Ivanova, E. A. Soboleva, E. V. Kulakova, V. P. Malyshev and I. V. Kirilyus, Russ. J. Appl. Chem., 2009, 82, 428.

50 V. L. Kornirnko, A. P. Tomilov, G. P. Vakar and N. V. Kalinichenko, Electrokhimiya, 1986, 22, 666.

51 S. E. Jeena, P. Gnanaprakasam and T. Selvaraju, Electroanalysis, 2016, 28, 1.

52 B. Liu, T. Wang, C. Yin and Z. Wei, J. Mater. Sci., 2014, 49, 5398.

53 J.-J. Lv, A.-J. Wang, X. Ma, R.-Y. Xiang, J.-R. Chen and J.-J. Feng, J. Mater. Chem. A, 2015, 3, 290.

54 O. Song-Il, J.-M. Yan, H.-L. Wang, Z.-L. Wang and Q. Jiang, Int. J. Hydrogen Energy, 2014, 39, 3755.

55 B. A. Kakade, H. Wang, T. Tamaki, H. Ohashi and T. Yamaguchi, RSC Adv., 2013, 3, 10487.

56 F. A. Wsrerhaus, R. V. Jagadeesh, G. Wienhöfer, M.-M. Pohl, J. Radnik, A.-E. Surkus, J. Rabeah, K. Junge, H. Junge, M. Nielsen, A. Buückner and M. Beller, Nat. Chem., 2013, $5,537$.

57 Y.-Y. Shen, Y. Sun, L.-N. Zhou, Y.-J. Li and E. S. Yeung, J. Mater. Chem. A, 2014, 2, 2977.

58 V. K. Gupta, N. Atar, M. L. Yola, Z. Üstündag and L. Uzun, Water Res., 2014, 48, 210.

59 G. Huang, L. Yang, X. Ma, J. Jiang, S.-H. Yu and H.-L. Jiang, Chem.-Eur. J., 2016, 22, 3470.

60 S. K. Srivastava, M. Guix and O. G. Schmidt, Nano Lett., 2016, 16, 817. 
61 W. Ye, J. Yu, Y. Zhou, D. Gao, D. Wang, C. Wang and D. Xue, Appl. Catal., B, 2016, 181, 371.

62 Y.-Y. Shen, Y. Sun, L.-N. Zhou, Y.-J. Li and E. S. Yeung, J. Mater. Chem. A, 2014, 2, 2977.
63 G. Cui, Z. Sun, H. Li, Z. Liu, Y. Tian and S. Yan, J. Mater. Chem. A, 2016, 4, 1771.

64 S. K. Ghosh, M. Mandal, S. Kundu, S. Nath and T. Pal, Appl. Catal., A, 2004, 268, 61. 